\title{
Time-Shift Homotopic Connectivity in Mesial Temporal Lobe Epilepsy
}

\author{
Q. Xu, Z. Zhang, W. Liao, L. Xiang, F. Yang, Z. Wang, G. Chen, Q. Tan, Q. Jiao, and G. Lu
}

\begin{abstract}
BACKGROUND AND PURPOSE: Voxel-mirrored intrinsic functional connectivity allows the depiction of interhemispheric homotopic connections in the human brain, whereas time-shift intrinsic functional connectivity allows the detection of the extent of brain injury by measuring hemodynamic properties. We combined time-shift voxel-mirrored homotopic connectivity analyses to investigate the alterations in homotopic connectivity in mesial temporal lobe epilepsy and assessed the value of applying this approach to epilepsy lateralization and the prediction of surgical outcomes in mesial temporal lobe epilepsy.
\end{abstract}

MATERIALS AND METHODS: Resting-state functional MR imaging data were acquired from patients with unilateral mesial temporal lobe epilepsy ( $n=62$ ) (31 left- and 31 right-side) and healthy controls $(n=33)$. Dynamic interhemispheric homotopic architecture seeding from each hemisphere was individually calculated by $0,1,2$, and 3 repetition time time-shift voxel-mirrored homotopic connectivity. Voxel-mirrored homotopic connectivity maps were compared between the patient and control groups by using 1-way ANOVA for each time-shift condition, separately. Group comparisons were further performed on the laterality of voxel-mirrored homotopic connectivity in each time-shift condition. Finally, we correlated the interhemispheric homotopic connection to the surgical outcomes in a portion of the patients $(n=20)$.

RESULTS: The patients with mesial temporal lobe epilepsy showed decreased homotopic connectivity in the mesial temporal structures, temporal pole, and striatum. Alterations of the bihemispheric homotopic connectivity were lateralized along with delays in the time-shift in mesial temporal lobe epilepsy. The patients with unsuccessful surgical outcomes presented larger interhemispheric voxel-mirrored homotopic connectivity differences.

CONCLUSIONS: This study showed whole patterns of dynamic alterations of interhemispheric homotopic connectivity in mesial temporal lobe epilepsy, extending the knowledge of abnormalities in interhemispheric connectivity in this condition. Time-shift voxelmirrored homotopic connectivity has the potential for lateralization of unilateral mesial temporal lobe epilepsy and may have the capability of predicting surgical outcomes in this condition.

ABBREVIATIONS: HC = healthy controls; mTLE = mesial temporal lobe epilepsy; ts = time-shift; VMHC = voxel-mirrored homotopic connectivity

nterhemispheric communication and coordination facilitate information processing in the human brain. ${ }^{1,2}$ Thus homotopic connections represent a fundamental characteristic of brain anat-

\footnotetext{
Received November 21, 2013; accepted after revision January 31, 2014. From the Departments of Medical Imaging (Q.X., Z.Z., W.L., L.X., Q.J., G.L.), Neurology (F.Y., G.C.), and Neurosurgery (Q.T.), Jinling Hospital, Nanjing University School of Medicine, Nanjing, China; Center for Cognition and Brain Disorders and the Affiliated Hospital (W.L.), Hangzhou Normal University, Hangzhou, China; Zhejiang Key Laboratory for Research in Assessment of Cognitive Impairments (W.L.), Hang zhou, China; Department of Medical Imaging (Z.W.), Nanjing Drum Tower Hospital, The Affiliated Hospital of Nanjing University Medical School, Nanjing, China; and Department of Medical Imaging (Q.J.), Taishan Medical College, TaiAn, China. This work was supported by the Natural Science Foundation of China (grant nos. 81271553, 81201155, 81201078, 81171328, 61131003, and 81020108022), Grants for Young Scholar of Jinling Hospital (grant nos. 2011060, 2011045, and 2011061), Chinese Key Grant (BWS11J063 and 10z026), and the China Postdoctoral Science Foundation (grant no. 2013M532229).
}

omy and function ${ }^{3,4}$ and have been considered an important indicator for depicting the physiologic and pathologic features of the brain. On the basis of resting-state functional MR imaging measurements, an approach based on voxel-mirrored homotopic connectivity (VMHC) quantifies the interhemispheric homotopic connections by measuring the functional connectivity between each voxel in 1 hemisphere and its mirrored counterpart. ${ }^{5}$ Zuo et $\mathrm{al}^{5}$ found age-related increases in interhemispheric functional connectivity in the primary sensorimotor areas and de-

Please address correspondence to Zhiqiang Zhang, MD, and Guangming Lu, MD, Department of Medical Imaging, Nanjing Jinling Hospital, 305\#, Eastern Zhongshan Rd, Nanjing 210002, China; e-mail: zhangzq2001@126.com (Z.Z.) or cjr.luguangming@ vip.163.com (G.L.)

- Indicates open access to non-subscribers at www.ajnr.org

三 Indicates article with supplemental on-line table.

http://dx.doi.org/10.3174/ajnr.A3934 
Demographic and clinical information of patients and healthy controls

\begin{tabular}{lccccccc}
\multicolumn{1}{c}{ Characteristic } & L-mTLE $(\boldsymbol{n}=31)$ & R-mTLE $(\boldsymbol{n}=31)$ & HC $(\boldsymbol{n}=33)$ & $\begin{array}{c}\text { ANOVA } \\
(\boldsymbol{P} \text { Value) }\end{array}$ & $\begin{array}{c}\text { L-mTLE vs R-mTLE } \\
(\boldsymbol{P} \text { Value })\end{array}$ & $\begin{array}{c}\text { L-mTLE vs HC } \\
(\boldsymbol{P} \text { Value })\end{array}$ & $\begin{array}{c}\text { R-mTLE vs HC } \\
(\boldsymbol{P} \text { Value })\end{array}$ \\
\hline Age (yr) & $26.5 \pm 8.4$ & $29.7 \pm 8.5$ & $26.7 \pm 7.3$ & $.231^{\mathrm{d}}$ & $.146^{\mathrm{c}}$ & $.903^{\mathrm{c}}$ & $.144^{\mathrm{c}}$ \\
Sex & $19 \mathrm{M} / 12 \mathrm{~F}$ & $16 \mathrm{M} / 15 \mathrm{~F}$ & $21 \mathrm{M} / 12 \mathrm{~F}$ & $.592^{\mathrm{b}}$ & $.442^{\mathrm{d}}$ & $.846^{\mathrm{d}}$ & $.330^{\mathrm{d}}$ \\
Successful outcome & 8 & 6 & $\mathrm{~N} / \mathrm{A}$ & $\mathrm{N} / \mathrm{A}$ & $\mathrm{N} / \mathrm{A}$ & $\mathrm{N} / \mathrm{A}$ & $\mathrm{N} / \mathrm{A}$ \\
Unsuccessful outcome & 4 & 2 & $\mathrm{~N} / \mathrm{A}$ & $\mathrm{N} / \mathrm{A}$ & $\mathrm{N} / \mathrm{A}$ & $\mathrm{N} / \mathrm{A}$ & $\mathrm{N} / \mathrm{A}$ \\
\hline
\end{tabular}

Note:-N/A indicates not applicable; L, left; R, right.

a One-way analysis of variance.

${ }^{\mathrm{b}}$ Kruskal-Wallis ANOVA.

'Two-sample $t$ test.

${ }^{\mathrm{d}} \chi^{2}$ test.

creases in the higher order processing areas, which provided insight into the evolution of brain development. Studies have also revealed specific alterations of homotopic connection in a cohort of brain diseases. ${ }^{6-8}$ Decreased VMHC in schizophrenia has been suggested to reflect the substantial impairment of interhemiespheric coordination in these patients. ${ }^{6}$ Anderson et $\mathrm{al}^{7}$ found homotopic connectivity alterations related to behavioral and developmental abnormalities in autism. ${ }^{7}$ More recently, studies have further correlated functional homotopic connectivity with microstructural impairment in multiple sclerosis ${ }^{8}$ and idiopathic generalized epilepsy. ${ }^{9}$

In contrast to the brain disorders featuring abnormal connection pathways as mentioned above, ${ }^{6,8}$ mesial temporal lobe epilepsy (mTLE) is a location-related disease characterized by hippocampal sclerosis. ${ }^{10}$ Unilateral mTLE can cause bilateral and distributed brain impairments due to seizure propagation via the mesial temporal epileptic network. ${ }^{11-13}$ Resting-state fMRI studies have shown asymmetric connections between bihemispheres ${ }^{14-16}$ and decreased connectivity between bilateral hippocampi in mTLE. ${ }^{17,18}$ These findings suggest that there are intraand interhemispheric connection abnormalities in unilateral mTLE. However, neither the homotopic alterations of wholebrain functional connectivity nor the relationship between asymmetric lesions and interhemispheric communication in this disease has been investigated.

fMRI-based VMHC provides a feasible way to observe the whole-brain homotopic connectivity alterations in mTLE. However, the conventional nondirectional functional connectivity measure, as used in VMHC, cannot detect the connection abnormalities resulting from deficits of the seed or target region. Recently, Lv et al ${ }^{19}$ proposed a time-shift (ts) analysis for restingstate functional connectivity. They quantified the temporal shift correlation between time courses of each voxel and global mean signal $^{19}$ and correlated the time shifts with the extent and degree of perfusion delay in patients with stroke. ${ }^{19,20}$ In addition, our previous study used time-shift correlation analysis to demonstrate the sequential effects of epileptic discharges on intrinsic network connectivity in children with absence epilepsy. ${ }^{21}$ Thus, time-shift delays in resting-state spontaneous connectivity were assumed to reflect brain hemodynamics and could measure the degree of brain injury; time-shift analysis also provides directional information as a measure of functional connectivity. ${ }^{21}$ In the current work, we combined the time-shift connectivity with the VMHC technique and applied them to resting-state fMRI data from patients with unilateral mTLE. We hypothesized that this strategy would allow us to assess the whole-brain homotopic con- nection impairments resulting from different hemispheres and may potentially be a tool for epileptic focus lateralization and surgical outcome prediction in mTLE.

\section{MATERIALS AND METHODS \\ Subjects}

Sixty-two patients with unilateral mTLE (31 left-sided and 31 right-sided) were recruited (demographic and clinical information are detailed in the Table). Part of the patient population (23 patients with left-sided mTLE) was reported in our previous work in which the Granger causal information of the hippocampus in left-sided mTLE was estimated. ${ }^{13}$ Diagnosis of mTLE was performed according to the International League Against Epilepsy 2001 classification based on a comprehensive evaluation, including seizure history and semiology, neurologic examination, diagnostic MR imaging, and electroencephalography records in all patients. Patients were selected on the basis of several criteria: 1) Patients who were young and middle-aged were included (patients younger than 18 years or older than 50 years were excluded); 2) patients who exhibited identifiable structural MR imaging abnormalities other than hippocampal sclerosis, such as cortical dysplasia, vascular malformation, or tumor were excluded. Twenty patients of the 62 underwent standard anterior temporal lobectomy: Fourteen cases ( 8 left- and 6 right-sided mTLE) had successful surgical outcomes, and 6 cases ( 4 left- and 2 right-sided mTLE) had unsuccessful outcomes. A successful outcome was defined as seizure-free with or without auras at the latest follow-up that occurred at least 1 year after the surgery, which satisfies the International League Against Epilepsy Outcome Criteria 1 or $2^{22}$ and Engel category $1 \mathrm{~A}$ or $1 \mathrm{~B} .{ }^{23}$ No postoperative MR imaging data were used in this article.

Moreover, healthy controls (HC) $(n=33)$ were recruited from the hospital staff. These controls did not have neurologic or psychiatric disorders at the time of the study. There were no differences in ages and sexes between the controls and patients (Table).

This study was approved by the local Medical Ethics Committee, and written informed consent was obtained from all the participants.

\section{Data Acquisition}

We acquired functional and structural images in patients and HC by using a Trio 3T scanner (Siemens, Erlangen, Germany). We used foam padding to minimize head motion. We acquired resting-state functional images by using a single-shot, gradient-recalled echo-planar imaging sequence ( 250 volumes, $\mathrm{TR}=2000$ $\mathrm{ms}, \mathrm{TE}=30 \mathrm{~ms}$, flip angle $=90^{\circ}, \mathrm{FOV}=240 \times 240 \mathrm{~mm}^{2}$, 
intersection gap $=0.4 \mathrm{~mm}$, voxel size $=3.75 \times 3.75 \times 4 \mathrm{~mm}^{3}, 30$ transverse sections aligned along the anterior/posterior commissure). Subjects were instructed simply to rest with their eyes closed, not to think about anything in particular, and not to fall asleep. Subsequently, we acquired high-resolution 3D T1weighted anatomic images in a sagittal orientation by using a

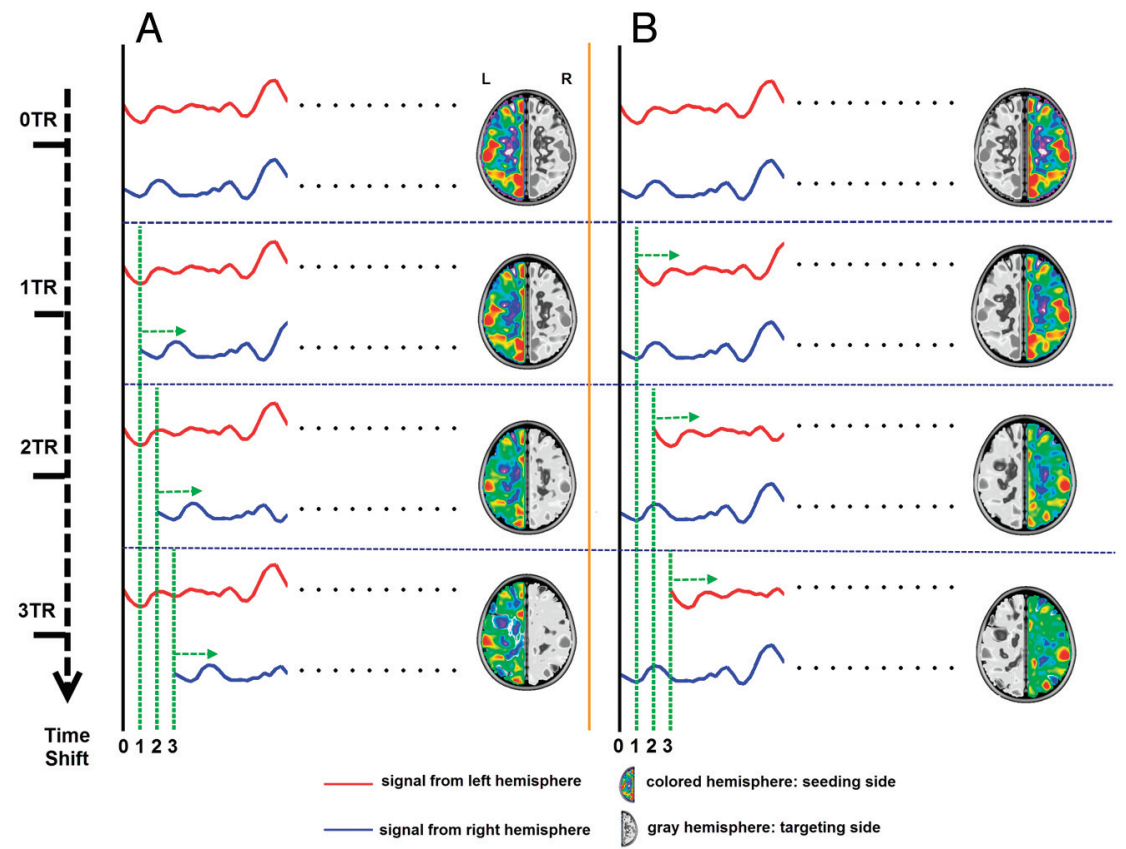

FIG 1. Workflow for time-shift voxel-mirrored homotopic connectivity calculation. A, Ts-VMHC of seeding from the left hemisphere. The left side was set as the seeding side; the right side, as the targeting side. The 0 TR, 1 TR, 2 TR, and 3 TR shifted time-courses of each voxel on the targeting side (blue line) were separately correlated with the time course of the mirrored voxel on the seeding side (red line), which generated 0 TR, 1 TR, 2 TR, and 3TR ts-VMHC hemispheric maps of the seeding (left) side. $B$, Similarly, 4 hemispheric ts-VMHC maps of the right side were also generated when taking the right hemisphere as the seeding side and the left hemisphere as the targeting side. magnetization-prepared rapid acquisition of gradient echo sequence $(\mathrm{TR}=$ $2300 \mathrm{~ms}, \mathrm{TE}=2.98 \mathrm{~ms}$, flip angle $=9^{\circ}$, FOV $=256 \times 256 \mathrm{~mm}^{2}$, voxel size $=1 \times$ $1 \times 1 \mathrm{~mm}^{3}, 176$ sections without intersection gap).

\section{Data Preprocessing}

Functional image preprocessing was performed by using the DPARSF 2.2 (http:// www.restfmri.net) and SPM8 (Wellcome Department of Imaging Neuroscience, London, UK; http://www.fil.ion.ucl.ac. $\mathrm{uk} / \mathrm{spm}$ ) toolkits. We excluded the first 10 images to ensure steady-state longitudinal magnetization, and then we corrected the remaining images for temporal differences and head motion. No translation or rotation parameters in any given dataset exceeded $\pm 1 \mathrm{~mm}$ or $\pm 1^{\circ}$. We then coregistered individual 3D T1 images to functional images. The 3D T1-weighted anatomic images were segmented into gray matter, white matter, and CSF by using unified segmentation. ${ }^{24}$ Then, a nonlinear spatial deformation was calculated from the gray matter images to a gray

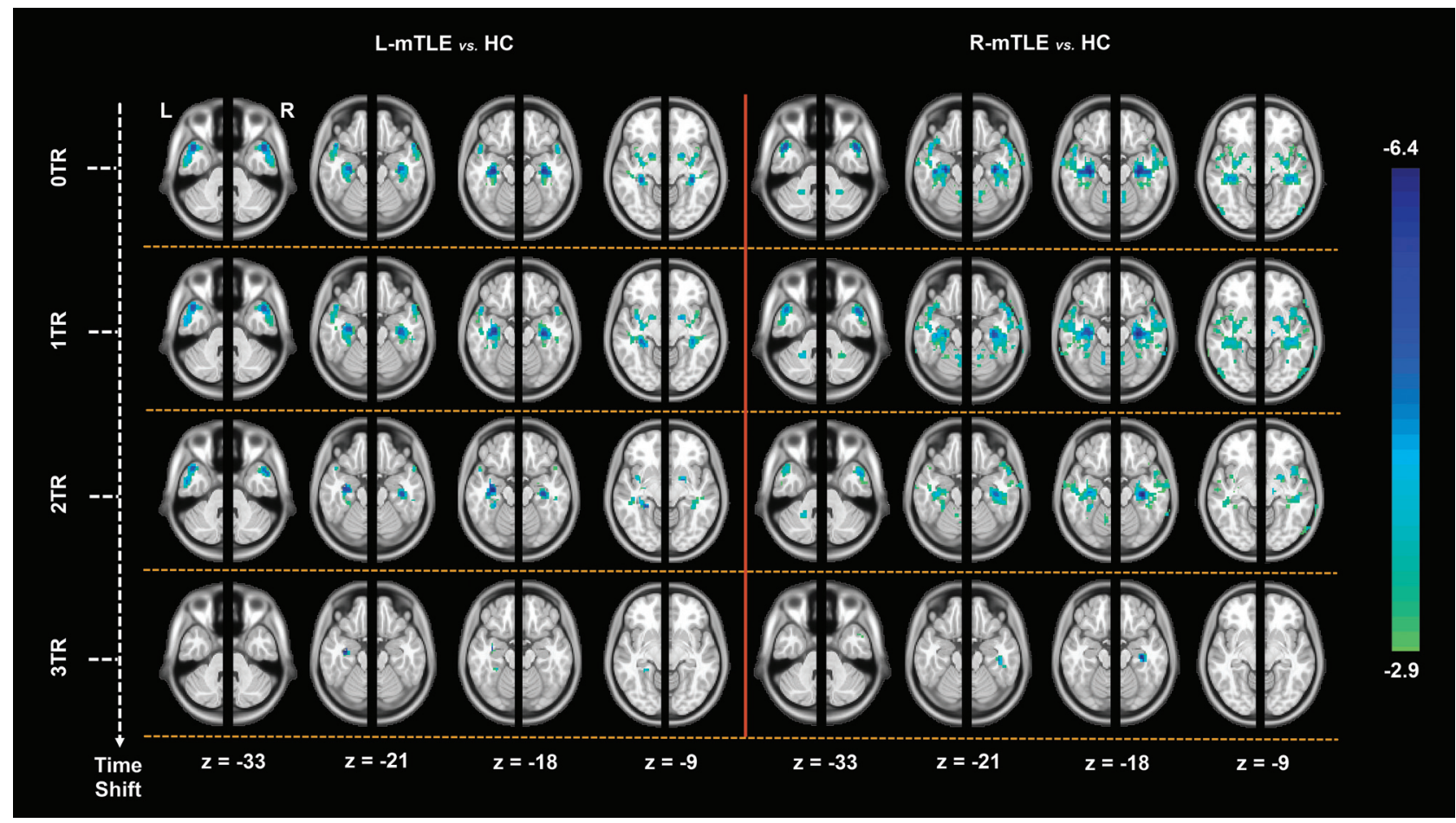

FIG 2. Group comparisons of ts-VMHC maps between the patients and healthy controls. The left and right hemispheric ts-VMHC maps (0 TR, 1 TR, 2 TR, and 3 TR) of each patient group were compared with those of the HC by using 2-sample $t$ tests $(P<.05$, AlphaSim corrected) under ANOVA. For a better visualization, we combined the comparison results of the left and right hemispheric ts-VMHC maps with a whole-brain map. The mesial temporal structures, limbic/paralimblic areas, and striatum areas show time-shift-dependent VMHC decreases. 

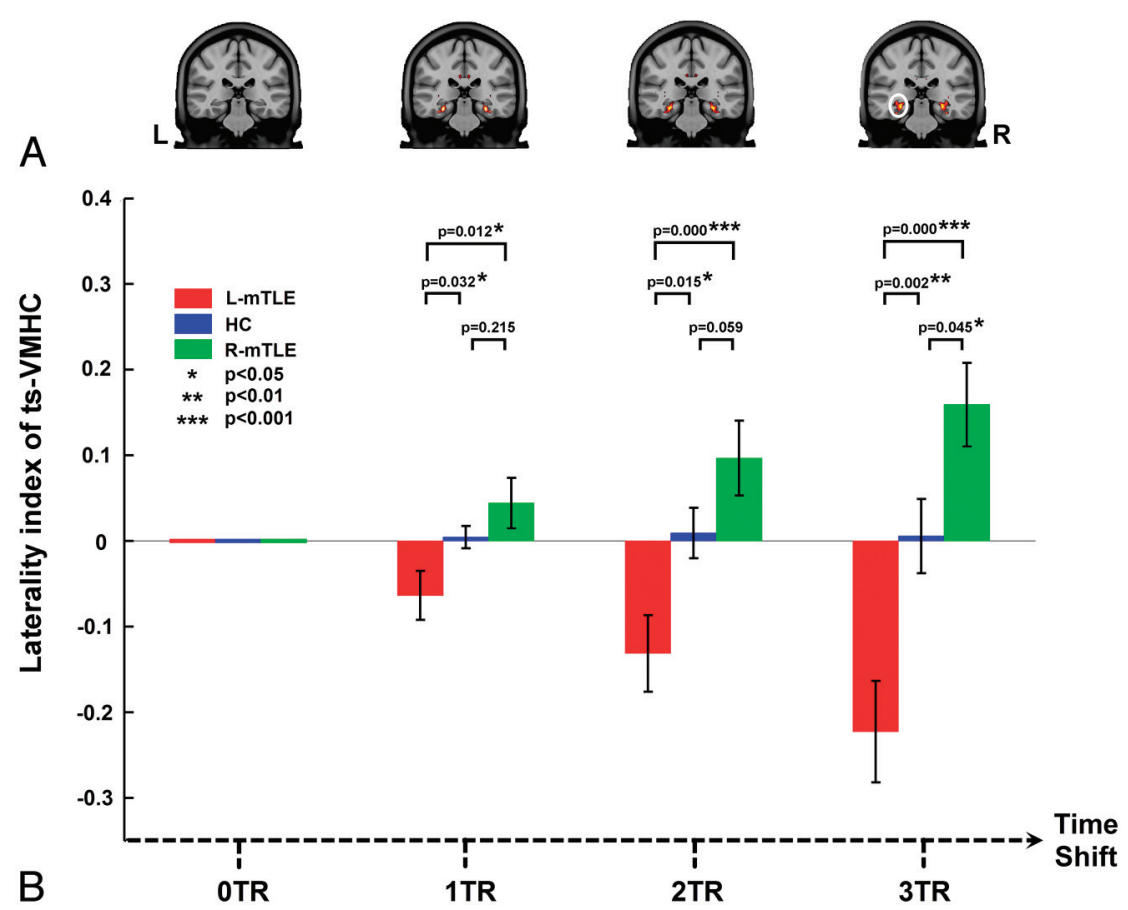

FIG 3. Laterality index analysis of ts-VMHC in mTLE. A, Group comparisons of voxelwise laterality maps of ts-VMHC. Along with the delay of time shifts, a more significant difference of VMHC laterality was found among the patient groups and healthy controls. $B$, Region of interest-based laterality analysis confirmed the above results. The region of interest was selected from the group comparing the result of voxelwise laterality map of ts-VMHC (white circle).

matter template in Montreal Neurological Institute space. This transformation was then applied to the functional images, which were resectioned at a resolution of $3 \times 3 \times 3 \mathrm{~mm}^{3}$. The data were bandpass-filtered $(0.01-0.08 \mathrm{~Hz})$. Linear regression was performed to remove the effect of 6 head-motion-averaged signals from the CSF, white matter, and the global brain signal. ${ }^{25}$ The residual images were further coregistered to a symmetric template according to previous studies. ${ }^{5,8}$ The symmetric template was created as follows: First, all participants' normalized gray matter images were averaged to create a mean normalized gray matter template. This template was then averaged with its left-right mirrored version to generate a groupspecific symmetric template. ${ }^{5}$ Finally, we spatially smoothed the images with an 8-mm full width at half maximum isotropic Gaussian kernel.

\section{Time-Shift Voxel-Mirrored Homotopic Connectivity}

We combined the time-shift analysis ${ }^{19}$ with voxel-mirrored homotopic connectivity analysis. ${ }^{5}$ We first took the left hemisphere as the seeding side and the right hemisphere as the targeting side. The 0 TR, 1 TR, 2 TR, and 3 TR shifted time-courses of each voxel on the targeting side were separately correlated with the time course of the mirrored voxel on the seeding side, which generated 0 TR, 1 TR, 2 TR, and 3 TR ts-VMHC hemispheric maps of the seeding (left) side. Similarly, 4 hemispheric ts-VMHC maps of the right side were also generated when taking the right hemisphere as the seeding side and the left hemisphere as the targeting side (Fig 1). The correlation maps were then converted to $z$ scores by using the Fisher r-to-z transformation.

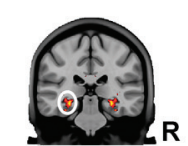

\section{Group Comparisons of ts-VMHC}

In the second-level analyses, the left and right hemispheric ts-VMHC maps ( $0 \mathrm{TR}$, $1 \mathrm{TR}, 2 \mathrm{TR}$, and $3 \mathrm{TR}$ ) of each patient group were compared with those of the HC by using a 2-sample $t$ test (AlphaSim corrected $P<.05$, combined height threshold $P<.01$, and extent threshold $\mathrm{k}>20$ voxels). For a better visualization, we combined the comparison results of the left and right hemispheric ts-VMHC maps with a whole-brain map ( Fig 2 and On-line Table).

\section{Laterality Analyses of ts-VMHC}

To quantitatively identify the difference between the left and right hemispheric tsVMHC and to further determine whether the ts-VMHC could be used for mTLE lateralization, we performed a voxelwise laterality analysis. In each individual of all 3 participant groups, the right hemispheric ts-VMHC map was subtracted from the left hemispheric ts-VMHC map and the results were divided by the summation of both maps. Then the laterality (hemispheric) maps of all 3 groups of participants were compared by using 1-way ANOVA implemented in SPM8 in each time-shift condition $(P<.05$, AlphaSim correction). Then, a post hoc region of interest-based analysis was subsequently performed. The ROIs were selected from the above ANOVA result in 3 TR time-shift conditions. The laterality values in the ROIs were extracted for ANOVA among the 3 participant groups (Fig 3).

\section{Predicting Surgical Outcomes of ts-VMHC}

We performed a preliminary analysis to retrospectively investigate the relationship between ts-VMHC and surgical outcomes of mTLE. Twenty patients who underwent anterior temporal lobectomy were included. The patients with right-sided mTLE (RmTLE) were left-right flipped to produce a homogeneous leftsided mTLE dataset. We calculated the difference of VMHC between bihemispheres by subtracting the connectivity values of the contralateral side from those of the lesional side (ie, the epileptogenic side). Subsequently, the interhemispheric differences were compared by using 2-sample $t$ tests between the patients with successful surgical outcomes and those with unsuccessful outcomes.

\section{RESULTS}

\section{Group Comparison Results of ts-VMHC}

On the basis of the comparison of the results of VMHC without time-shift (ie, 0 TR shift), both patient groups showed decreased homotopic connectivity in the regions of limbic/paralimbic areas (mainly in the mesial temporal structures including the hippocampus/parahippocampus, temporal pole, amygdala, and insula) and striatum areas (mainly in the putamen and pallidum) relative to the $\mathrm{HC}(P<.05$ AlphaSim correction). Along with the 


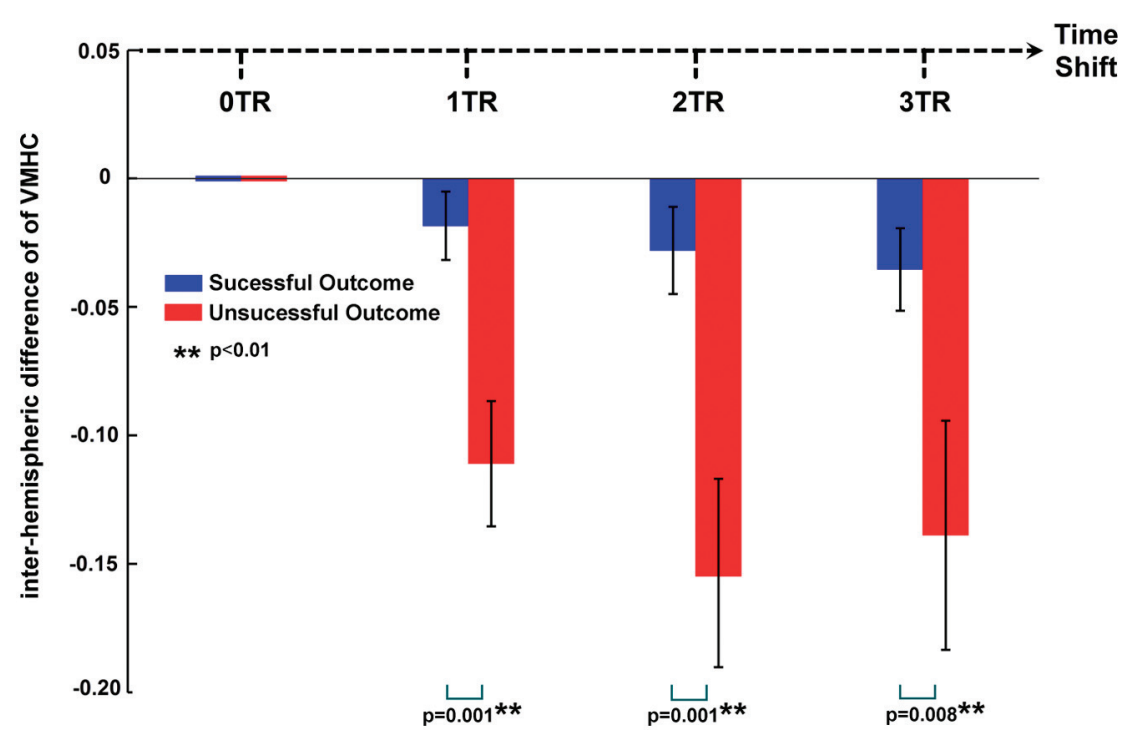

FIG 4. Ts-VMHC and prediction of surgical outcomes of mTLE. The interhemispheric VMHC differences were compared by using 2-sample $t$ tests between the patients with successful surgical outcomes and those with unsuccessful outcomes. The patients with unsuccessful surgical outcomes had larger differences than those with successful outcomes at each time-shift condition. Moreover, along with the time shifts, the interhemispheric VMHC differences increased in both the groups.

time-shift delays, the decreases in homotopic connectivity in both hemispheres tended to be weaker, whereas the decreases on the lesioned side (ie, the left side in the left-sided mTLE and the right side in the right-sided mTLE patients) were more persistent. Until the 3 TR shift, only the difference in the mesial temporal structures of the lesioned side survived. No increase in homotopic connectivity was detected in the patient groups (On-line Table and Fig 2).

\section{Laterality Analyses of ts-VMHC}

Along with the time-shift delays at $1 \mathrm{TR}, 2 \mathrm{TR}$, and $3 \mathrm{TR}$, the results of the 1-way ANOVA among subject groups showed more significant laterality of homotopic connectivity in the mesial temporal structures. Post hoc region-of-interest analyses repeated the above 3 TR time-shifted ANOVA results (Fig 3). Along with time shifts of 1 TR, 2 TR, and 3 TR, the difference between the patients with left-sided mTLE and those with right-sided mTLE became more significant $(P=.001$ in $1 \mathrm{TR}, P<.001$ in $2 \mathrm{TR}$, and $P<.001$ in 3TR).

\section{Predicting Surgical Outcomes}

The preliminary analysis correlating ts-VMHC with surgical outcomes showed that the patients with unsuccessful surgical outcomes had larger VMHC differences between the hemispheres than those with successful outcomes at each time-shift condition (1 TR-shifted, $P=.001 ; 2$ TR-shifted, $P=.001 ; 3$ TR-shifted, $P=$ .008). Moreover, along with time shifts of 1 TR, 2 TR, and 3 TR, the interhemispheric VMHC differences increased in both the groups with successful and unsuccessful outcomes (Fig 4).

\section{DISCUSSION}

In this study, we innovatively combined time-shift functional connectivity analysis with VMHC analysis and applied them to data from patients with unilateral mTLE. First, our results showed decreased homotopic connectivity in the mesial temporal structures, temporal pole, and striatum in mTLE, which provided the first complete picture of interhemispheric connectivity abnormalities in mTLE. Second, we found that the alterations in the bihemispheric homotopic connectivity led to lateralization along with time-shift delays in patients with mTLE. This finding suggests that tsVMHC may have the potential for lateralization of unilateral mTLE. Third, the hemispheric differences in homotopic connectivity were related to surgical outcomes in patients with mTLE, which indicates that the time-shift homotopic connectivity may be predictive of surgical outcomes in mTLE.

We first revealed the whole-brain patterns of altered homotopic connectivity in mTLE. We found that the mesial temporal structures, including the hippocampus and parahippocampal gyrus, showed decreased homotopic connectivity, consistent with previous studies. ${ }^{14,15,17}$ Furthermore, we found decreased homotopic connectivity in the temporal pole, amygdala, insula, putamen, and pallidum. These regions have been regarded as key nodes in the mesial temporal epileptic networks and play important roles in the generation, propagation, and modulation of epileptic activity. ${ }^{14,26}$ Imaging studies have demonstrated structural ${ }^{27,28}$ and functional abnormalities in these regions. ${ }^{29-33}$ Thus, we considered that reduced homotopic connectivity might result from nodal impairments of these regions in mTLE. Moreover, studies have proposed a variety of propagation patterns of epileptic activity from the originating focus to the contralateral side; the limbic structures and basal ganglia are the important nodes within the propagation pathway. ${ }^{34,35}$ Here we cautiously proposed another explanation, that time-shift-dependent alterations of VMHC of these regions might represent propagations of epileptic activity.

Along with the time-shift delays, the decreases of the bihemispheric homotopic connectivity were more lateralized in patients with unilateral mTLE. The lesioned side showed more persistent decrease in homotopic connectivity. ${ }^{5}$ We introduced a time-shift functional connectivity analysis, different from the conventional VMHC approach, which provided directional information to identify connectivity abnormalities resulting from different nodal impairments. Time-shift functional connectivity was first proposed by $\mathrm{Lv}$ et $\mathrm{al}^{19}$ in their fMRI study on stroke. They proposed that the time-shift delays in resting-state fMRI connectivity represent the hemodynamics/perfusion property of stroke and could be used to measure the extent of the brain lesions. ${ }^{19,20}$ Here, we assume that different decreases in the time-shift homotopic connectivity between the hemispheres may result from different hemodynamic properties between the hemispheres in mTLE. Previous studies showed that the epileptic focus presented interictal hypoperfusion in mTLE. ${ }^{36,37}$ This finding confirmed our hypothesis that the strategy of combining time-shift functional connectivity with VMHC had the potential to lateralize unilateral mTLE. 
Our preliminary analysis showed that the results of ts-VMHC could be related to the surgical outcomes in patients with mTLE. The patients with unsuccessful outcomes had more interhemispheric VMHC differences. The finding suggested that the patients with unsuccessful outcomes might have more severe lesions and presented more disruption of interhemispheric communication. Previous studies have shown that resting-state fMRI has the capability of predicting surgical outcomes in mTLE. ${ }^{16,38}$ Different from those studies that used predefined region-of-interest analysis, ${ }^{16,38}$ our study used an unbiased voxelwise analysis approach and simultaneously observed the dynamic property of intrinsic connectivity in mTLE. Our study provided more comprehensive information for resting-state fMRI in the prediction of surgical outcome in mTLE.

\section{Limitations}

A few of the limitations in this study are noteworthy. First, we did not address the possible effects of antiepileptic drugs on fMRI in the study patients. Second, another presumption of time-shift connectivity might reflect propagation of epileptic activation; simultaneous electroencephalography and fMRI to record interictal epileptic discharges are needed in future studies. Third, the included patients had detectable hippocampal sclerosis, which might weaken the significance of epilepsy lateralization. Future study on patients with negative MR imaging findings is needed to validate this study. Finally, this work only observed the interhemispheric homotopic connectivity; measures of intrahemispheric and whole-brain connectivity might benefit from the understanding of the underlying mechanisms of connectivity alterations in epilepsy.

\section{CONCLUSIONS}

This study combined time-shift functional connectivity analysis with VMHC analysis and observed ts-VMHC alteration in patients with mTLE. The results showed the whole-brain pattern of aberrant interhemispheric connectivity in MTLE and further implicated the associated nodal impairment. Moreover, our study provided a novel fMRI approach that has potential value for epileptic focus lateralization and predicting the surgical outcome of mTLE.

\section{REFERENCES}

1. Hervé PY, Zago L, Petit L, et al. Revisiting human hemispheric specialization with neuroimaging. Trends Cogn Sci 2013;17:69-80

2. Doron KW, Bassett DS, Gazzaniga MS. Dynamic network structure of interhemispheric coordination. Proc Natl Acad Sci $U S A$ 2012;109:18661-68

3. Stark DE, Margulies DS, Shehzad ZE, et al. Regional variation in interhemispheric coordination of intrinsic hemodynamic fluctuations. J Neurosci 2008;28:13754-64

4. Jo HJ, Saad ZS, Gotts SJ, et al. Quantifying agreement between anatomical and functional interhemispheric correspondences in the resting brain. PloS One 2012;7:e48847

5. Zuo XN, Kelly C, Di Martino A, et al. Growing together and growing apart: regional and sex differences in the lifespan developmental trajectories of functional homotopy. J Neurosci 2010;30:15034-43

6. Hoptman MJ, Zuo X-N, D'Angelo D, et al. Decreased interhemispheric coordination in schizophrenia: a resting state fMRI study. Schizophr Res 2012;141:1-7

7. Anderson JS, Druzgal TJ, Froehlich A, et al. Decreased interhemi- spheric functional connectivity in autism. Cereb Cortex 2011;21: 1134-46

8. Zhou Y, Milham M, Zuo XN, et al. Functional homotopic changes in multiple sclerosis with resting-state functional MR imaging. AJNR Am J Neuroradiol 2013;34:1180-87

9. Ji GJ, Zhang Z, Xu Q, et al. Generalized tonic-clonic seizures: aberrant interhemispheric functional and anatomical connectivity. $R a$ diology 2014;271:839-47

10. Wieser HG. ILAE commission report: mesial temporal lobe epilepsy with hippocampal sclerosis. Epilepsia 2004;45:695-714

11. Spencer SS. Neural networks in human epilepsy: evidence of and implications for treatment. Epilepsia 2002;43:219-27

12. Engel J. Mesial temporal lobe epilepsy: what have we learned? Neuroscientist 2001;7:340-52

13. Ji GJ, Zhang Z, Zhang H, et al. Disrupted causal connectivity in mesial temporal lobe epilepsy. PloS One 2013;8:e63183

14. Bettus G, Guedj E, Joyeux F, et al. Decreased basal fMRI functional connectivity in epileptogenic networks and contralateral compensatory mechanisms. Hum Brain Mapp 2009;30:1580-91

15. Morgan VL, Rogers BP, Sonmezturk HH, et al. Cross hippocampal influence in mesial temporal lobe epilepsy measured with high temporal resolution functional magnetic resonance imaging. Epilepsia 2011;52:1741-49

16. Bettus G, Bartolomei F, Confort-Gouny S, et al. Role of resting state functional connectivity MRI in presurgical investigation of mesial temporal lobe epilepsy. J Neurol Neurosurg Psychiatry 2010;81: 1147-54

17. Pereira FR, Alessio A, Sercheli MS, et al. Asymmetrical hippocampal connectivity in mesial temporal lobe epilepsy: evidence from resting state fMRI. BMC Neurosci 2010;11:66

18. Pittau F, Grova C, Moeller F, et al. Patterns of altered functional connectivity in mesial temporal lobe epilepsy. Epilepsia 2012;53: 1013-23

19. Lv Y, Margulies DS, Cameron Craddock R, et al. Identifying the perfusion deficit in acute stroke with resting-state functional magnetic resonance imaging. Ann Neurol 2013;73:136-40

20. Amemiya S, Kunimatsu A, Saito N, et al. Cerebral hemodynamic impairment: assessment with resting-state functional MR imaging. Radiology 2014;270:548-55

21. Zhang Z, Liao W, Wang Z, et al. Epileptic discharges specifically affect intrinsic connectivity networks during absence seizures. J Neurol Sci 2014;336:138-45

22. Wieser H, Blume W, Fish D, et al, for the Commission on Neurosurgery of the International League Against Epilepsy (ILAE). ILAE Commission Report: proposal for a new classification of outcome with respect to epileptic seizures following epilepsy surgery. Epilepsia 2001;42:282-86

23. Engel J Jr. Update on surgical treatment of the epilepsies: summary of The Second International Palm Desert Conference on the Surgical Treatment of the Epilepsies (1992). Neurology 1993;43:1612-17

24. Ashburner J, Friston KJ. Unified segmentation. Neuroimage 2005; 26:839-51

25. Fox MD, Snyder AZ, Vincent JL, et al. The human brain is intrinsically organized into dynamic, anticorrelated functional networks. Proc Natl Acad Sci U S A 2005;102:9673-78

26. Bertram EH, Zhang DX, Mangan P, et al. Functional anatomy of limbic epilepsy: a proposal for central synchronization of a diffusely hyperexcitable network. Epilepsy Res 1998;32:194-205

27. Mueller SG, Laxer KD, Cashdollar N, et al. Voxel-based optimized morphometry (VBM) of gray and white matter in temporal lobe epilepsy (TLE) with and without mesial temporal sclerosis. Epilepsia 2006;47:900-07

28. Li J, Zhang Z, Shang H. A meta-analysis of voxel-based morphometry studies on unilateral refractory temporal lobe epilepsy. Epilepsy Res 2012;98:97-103

29. Jafari-Khouzani K, Elisevich K, Karvelis KC, et al. Quantitative multi-compartmental SPECT image analysis for lateralization of temporal lobe epilepsy. Epilepsy Res 2011;95:35-50 
30. Kim BJ, Hong SB, Seo DW. Differences in ictal hyperperfusion of limbic-related structures between mesial temporal and neocortical epilepsy. Epilepsy Res 2008;81:167-75

31. Semah F, Baulac M, Hasboun D, et al. Is interictal temporal hypometabolism related to mesial temporal sclerosis? A positron emission tomography/magnetic resonance imaging confrontation. Epilepsia 1995;36:447-56

32. Zhang Z, Lu G, Zhong Y, et al. fMRI study of mesial temporal lobe epilepsy using amplitude of low-frequency fluctuation analysis. Hum Brain Mapp 2010;31:1851-61

33. Liao W, Zhang Z, Pan Z, et al. Altered functional connectivity and small-world in mesial temporal lobe epilepsy. PloS One 2010; 5:e8525

34. Kücker S, Töllner K, Piechotta M, et al. Kindling as a model of tem- poral lobe epilepsy induces bilateral changes in spontaneous striatal activity. Neurobiol Dis 2010;37:661-72

35. Pan J, Spencer D, Kuzniecky R, et al. Metabolic networks in epilepsy by MR spectroscopic imaging. Acta Neurol Scand 2012;126:411-20

36. Tae WS, Joo EY, Kim JH, et al. Cerebral perfusion changes in mesial temporal lobe epilepsy: SPM analysis of ictal and interictal SPECT. Neuroimage 2005;24:101-10

37. Marques LH, Ferraz-Filho JR, Lins-Filho ML, et al. Interictal SPECT in the presurgical evaluation in epileptic patients with normal MRI or bilateral mesial temporal sclerosis. Arq Neuropsiquiatr 2009;67: $639-42$

38. Negishi M, Martuzzi R, Novotny EJ, et al. Functional MRI connectivity as a predictor of the surgical outcome of epilepsy. Epilepsia 2011;52:1733-40 